Folia Primatol 1995;64:232

\title{
Subject Index Vol. 64, 1995
}

Active phase 167 Adaptation 1 Agonistic buffering 153 Allomothering 62 Allonursing 62 Alouatta caraya 55

seniculus 158 Angolan black-and-white colobus 196 Antipronograde primates 218 Aotus 107

Bioko Island 49

Biomechanics 1

Bipedalism 1

Black mangabey 196

Black-cheeked white-nosed guenon 196

Blue monkey 69

Bone remodelling 1

Brazil 55

Bridging behaviour 153

'Brotherhoods' 207

Budongo Forest Reserve 69

Callimico 107

goeldii 192 Callithrix 107

geoffroyi 163 Callitrichidae 140,180 Cameroon 62 Captive groups 192 Capuchin monkeys 158

Cebidae 55 Cebuella 107 Cebus albifrons 158 Cercocebus 196 Cercopithecine monkeys 49

Cercopithecus 196

erythrotis 49 mitis 69 nictitans 49

- pogonias 49

Chimpanzees 44

Climbing behaviour 218

Coercion defence 207

Cognitive abilities 44

Colobus 196

Common marmoset 180

Communication 201

Competition 132

Conservation 49, 158

Consortship 153

Copper 215

Copulation 132

Copulatory call 132

frequency 146

de Brazza's monkey 196 Dental microwear 30 Dentition 215 Diet 167 Discrete traits 37

Dispersal 192 Drills 49

Ejaculation frequency 146 Electromyography 218 Energy-dispersive X-ray microanalysis

30 Erythrocebus patas 62 Eye preference 180 
Felis wiedii 163 Female bonding 207

choice 207

competition 192

mating patterns 225 Field study 69 Foraging synchrony 167 Frugivory 124 Functional morphology 1

Geoffrey’s marmoset 163 Goeldi’s monkey 192 Gorilla gorilla 37 Group composition 192

- $\quad$ size 158,167

Guenons 49, 69, 196

Habitat disruption 124 Hand preference 180 Handedness 180 'Hired-gun’ principle 207

Homeoboxgene 37 Howler monkey 55

Infant carriage 153 desertion 62

distress calls 201

kidnapping 62 Infanticide 69 Interference 132 Intragroup competition 167

Japanese macaque 1,132,218

Kin selection 207

Lead 215 Leaves 30 Leontopithecus 107 Locomotion 55, 218

Macacafascicularis 30

- $\quad$ fuscata 1, 132, 218

- $\quad$ - yakui 167

mulatía 215

thibetana 153

Macaque(s) 30, 153, 201, 215

Madagascar 124

Male aggression 207

intersexual coercion 207 intrasexual competition 207

Mandrillus leucophaeus 49

Margaycat 163

Mate choice 132

Maternal responsiveness 201

Mating behaviour 146 systems 225

Meat eating 69

Mobbing 163 Mona monkey 196 Mother-infant interaction 44 Mouth use 180

New World monkeys 107 Novel stimuli 44

Occipital vertebra 37 Oestrogen 107

excretion 140 Old World monkeys 196 Ovarian cycle 107,140

Pan troglodytes 44 Patas monkeys 62 Phytogeny 107 Population density 158 Postcranial skeleton

1 Predator 163 Primates 30

Processus paracondylaris 37 Pronograde primates 218

Quadrupedalism 55

Ranging 167 Reciprocal altruism 207 Red howlers 158 Red-bellied tamarins 140 Relative

abundance 49 Reproduction 107 Reproductive competition 146

- $\quad$ suppression 140

Ruffed lemurs 124

Saguinus 107

labiatus 140 Selective logging 124 Sex ratio 158 Sexual behavio(u)r 132,146

dimorphism 207 
- $\quad$ selection 146,207

Silica 30

Size classes 55 Social cognition 153 referencing 44

- $\quad$ tool 153

Sociality 207

Sperm competition 146,225

- $\quad$ length 225

Spina ligamenti apicis dentis 37

Terrestrial locomotion 55 Testes size 225 Trace elements 215 Triadic interaction 153 Trinidad 158

Urinary steroid metabolites 107

Varecia 124 Vocalization 132

Zaire 196

232 